\title{
Pump-Probe Photoionization Study of the Passage and Bifurcation of a Quantum Wave Packet Across an Avoided Crossing
}

\author{
Yasuki Arasaki and Kazuo Takatsuka* \\ Department of Basic Science, Graduate School of Arts and Sciences, University of Tokyo, Komaba, 153-8902, Tokyo, Japan
}

Kwanghsi Wang and Vincent McKoy

Laboratory for Molecular Sciences, California Institute of Technology, Pasadena, California 91125, USA

(Received 26 November 2002; published 19 June 2003)

\begin{abstract}
The application of femtosecond pump-probe photoelectron spectroscopy to directly observe vibrational wave packets passing through an avoided crossing is investigated using quantum wave packet dynamics calculations. Transfer of the vibrational wave packet between diabatic electronic surfaces, bifurcation of the wave packet, and wave packet construction via nonadiabatic mixing are shown to be observable as time-dependent splittings of peaks in the photoelectron spectra.
\end{abstract}

DOI: 10.1103/PhysRevLett.90.248303

The concept of nonadiabatic transitions is fundamental to an understanding of chemical phenomena [1,2]. A typical example, and the one of interest here, is intramolecular electron transfer induced by vibrational motion in the excited state of alkali halides such as the $\mathrm{NaI}$ molecule. Pump-probe studies of this system have demonstrated the decrease of wave packet population on the excited adiabatic surface due to dissociation at the avoided crossing $[3,4]$. Oscillations in the population of the dissociative products ( $\mathrm{Na}$ and $\mathrm{I}$ ) due to the interference of wave packets on the covalent and ionic potentials merging at the avoided crossing have also been observed [3]. Although bifurcation of wave packets must be invoked to explain these observations, no real-time evidence of the instance of wave packet bifurcation has yet been experimentally observed. Such direct observation, if possible, would be interesting in itself, but also quite significant to studies of electron transfer, wave packet engineering, and reaction control through wave packet splitting and mixing. Also, from the perspective of quantum measurement, wave packet bifurcation corresponding to an intramolecular double-slit experiment will shed light on the evolution of quantum entanglement between electronic and nuclear motion [5]. Bifurcation and merging of wave packets are important as an intrinsic mechanism of "quantum chaos," which has no simple classical counterpart [6-8].

Pump-probe photoelectron spectroscopy has been demonstrated to be a powerful means to monitor real-time reaction dynamics [9-14]. We here show theoretically that the method permits real-time observations of a wave packet passing through an avoided crossing, using excited-state dynamics of $\mathrm{NaI}$ as an example.

The pump-probe spectroscopy of $\mathrm{NaI}$ has been studied extensively [3,4,15-21]. Figure 1 depicts the pump-probe scheme and the relevant potential curves for femtosecond photoelectron spectroscopy of the excited-state wave packet dynamics. The adiabatic excited state features an
PACS numbers: 82.20.Gk, 33.60.-q, 82.53.Eb, 82.53.Kp

extended well whose character changes from covalent at shorter distances to ionic at larger distances due to an avoided crossing with the ground state at $\sim 7 \AA[22,23]$. In the diabatic representation, the same system is viewed as an ionic curve (here called $V_{1}$ ) intersecting a covalent curve $\left(V_{2}\right)$, with an associated nonadiabatic interaction $\left(V_{12}\right)$. The diabatic curves are shown in Fig. 1.

The excited-state wave packet dynamics to be investigated are shown as contour plots of the square modulus of the time-evolving wave packets in Fig. 2. Centered at time $t=0$, the pump pulse [ $\hbar \omega_{1}=3.73 \mathrm{eV}$, full width at half maximum (FWHM) $40 \mathrm{fs}$ ] forms a wave packet on the excited electronic state directly above the initial ground state wave packet at $2.7 \AA$. The wave packet on the $V_{2}$ curve reaches the crossing point (depicted here with dotted lines) at about 0.2 ps. Bifurcation of the wave packet occurs at this point; most of the packet $(90 \%)$ is transferred onto the $V_{1}$ curve (i.e., the evolution is mostly adiabatic), while a small portion remains on the $V_{2}$ curve and eventually dissociates. The packet reflected from the

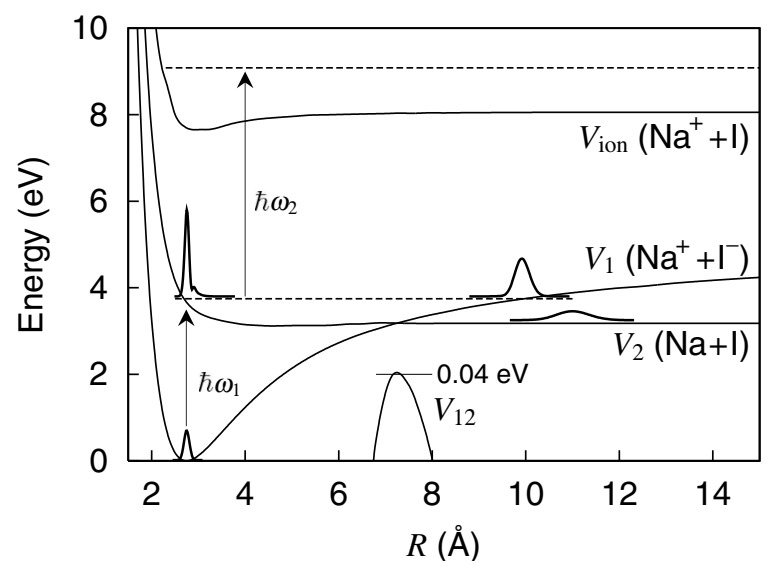

FIG. 1. Diabatic potential curves and the pump-probe scheme. 


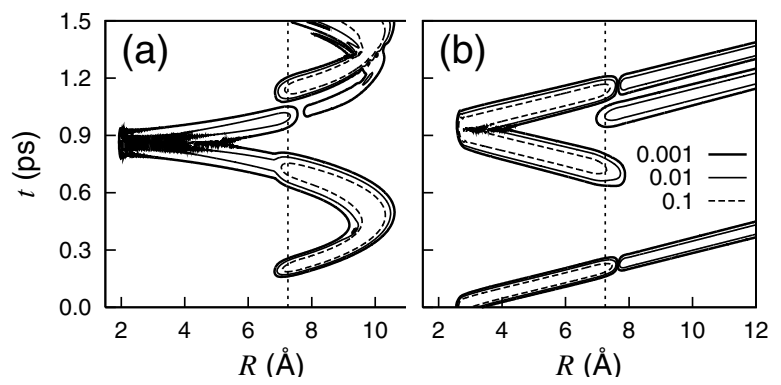

FIG. 2. Contour plots of wave packet propagation on (a) the diabatic $V_{1}$ curve and (b) the $V_{2}$ curve. For clarity, the wave packet component on $V_{1}$ immediately after the pump pulse is not shown.

right-hand turning point on $V_{1}$ undergoes a second bifurcation at about $0.7 \mathrm{ps}$ as it moves inwards. The wave packets thus bifurcated onto the two curves have generally different periods for returning to the crossing point. The larger component on $V_{2}$ hits the inner wall and returns to the nonadiabatic region by $1.1 \mathrm{ps}$, while the smaller component on $V_{1}$ moves faster in the steep well and thus arrives back in the nonadiabatic region by $1.0 \mathrm{ps}$.

We compute the photoelectron spectra using a quantum wave packet method described in a previous paper [13] with enhancements to handle the nonadiabatic interactions. Briefly, the system is expanded in terms of the three relevant electronic states: $\Phi_{1}\left(V_{1}\right.$ state $), \Phi_{2}\left(V_{2}\right.$ state $)$, and $\Phi_{\mathbf{k}}^{(-)}$(ion state), as

$$
\begin{aligned}
\Psi(\mathbf{r}, R, t)= & \chi_{1}(R, t) \Phi_{1}(\mathbf{r} ; R)+\chi_{2}(R, t) \Phi_{2}(\mathbf{r} ; R) \\
& +\int d \mathbf{k} \chi_{\mathbf{k}}(R, t) \Phi_{\mathbf{k}}^{(-)}(\mathbf{r} ; R),
\end{aligned}
$$

where $\mathbf{k}$ labels the wave vector of the photoelectron. Substitution into the time-dependent Schrödinger equation results in coupled dynamical equations for the vibrational wave packets $\chi_{1}, \chi_{2}$, and $\chi_{\mathbf{k}}$ of the form (in atomic units)

$$
\begin{aligned}
i \frac{\partial}{\partial t} \chi_{1}(R, t)=[ & \left.T_{\mathrm{N}}+V_{1}(R)\right] \chi_{1}+V_{12}(R) \chi_{2} \\
& +\int d \mathbf{k} V_{1, \mathbf{k}}\left(\mathbf{R}, \theta_{P}, \mathbf{k}, t\right) \chi_{\mathbf{k}}, \\
i \frac{\partial}{\partial t} \chi_{\mathbf{k}}(R, t)= & {\left[T_{\mathrm{N}}+V_{\text {ion }}(R)+\frac{k_{j}^{2}}{2}\right] \chi_{\mathbf{k}} } \\
& +\sum_{d=1,2} V_{\mathbf{k}, d}\left(\mathbf{R}, \theta_{P}, \mathbf{k}, t\right) \chi_{d},
\end{aligned}
$$

where $T_{\mathrm{N}}$ is the kinetic energy operator; $V_{\mathbf{k}, d}$ is the ionization interaction matrix element, including dependence on molecular geometry $(\mathbf{R})$, photoelectron energy and angle, probe pulse shape, and polarization $\left(\theta_{P}\right)$; and molecular rotation has been neglected. The equation for $\chi_{2}$ is analogous to that for $\chi_{1}$. The photoelectron spectra are calculated from the vibrational wave packets after the probe interaction as

$$
P\left(\varepsilon_{k}\right)=k \int d R d \hat{k}\left|\chi_{\mathbf{k}}(R)\right|^{2}
$$

The photoionization matrix elements employed in these studies are obtained using high-quality wave functions for the bound states and photoelectron continuum. Further details of the calculation will be published in a later paper [24]. Preliminary results for this calculation were presented earlier [21].

A summary of some key features of the NaI wave packet dynamics and the photoionization scheme is as follows. Transfer of the vibrational wave packet from the "flat" $V_{2}$ curve to the "steep" $V_{1}$ curve results not only in a rapid change in kinetic energy but also in a drastic change in the ionization amplitude, the ionization amplitude from the ionic $V_{1}$ state being much larger than that from the covalent $V_{2}$ state. The amplitude difference is dependent on the orientation of the probe pulse's polarization vector relative to the molecular axis and is greatest when the molecule is perpendicular to the probe polarization. The results presented here assume that arrangement. Wave packet motion is monitored by a timedelayed ionizing probe pulse $\left(\hbar \omega_{2}=5.34 \mathrm{eV}\right.$, FWHM $=$ $40 \mathrm{fs}$ ). With the pump and probe energies fixed, the Condon principle (which we have confirmed to be mostly valid in our system) implies that the kinetic energy of the photoelectrons is essentially determined by the kinetic energy of the relevant vibrational wave packet. Ionization from the flat region of the $V_{2}$ curve beyond $\sim 5 \AA$ produces a distribution of photoelectrons centered at $\sim 0.5 \mathrm{eV}$, whereas ionization from the $V_{1}$ curve to the right of the crossing produces higher photoelectron energies, typically in the range of $0.6-1.0 \mathrm{eV}$. In particular, ionization at the right-hand turning point yields photoelectrons with $\sim 1.0 \mathrm{eV}$. The $5.34 \mathrm{eV}$ probe pulse can also ionize wave packets off the $V_{1}$ curve below and to the left of the crossing point down to $\sim 6 \AA$, which typically gives a peak at $\sim 0.2 \mathrm{eV}$ in the photoelectron signal. Although ionization from $V_{2}$ to the right of the crossing point is energetically possible, the small population produced there, along with the small photoionization amplitude, results in a negligible signal. The features just described should be more or less common to systems of intramolecular electron transfer.

In what follows, we demonstrate via three examples that patterns of splitting in the photoelectron signal can directly reflect the passage through and bifurcation of the wave packet at the crossing point. Photoelectron spectra from the first nonadiabatic passage are shown in Fig. 3. Figure 3(a) shows an enlarged view of the diabatic potential curves near the crossing; overlaid are the modulus squared of wave packets at three representative times. Wave packets drawn with solid lines are on the $V_{1}$ curve, those with broken lines on $V_{2}$. Figure 3(b) shows the photoelectron spectra for the same snapshot times as (a). At $190 \mathrm{fs}$, the excited-state wave packet is mostly on the $V_{2}$ curve, resulting in photoelectron kinetic energies 

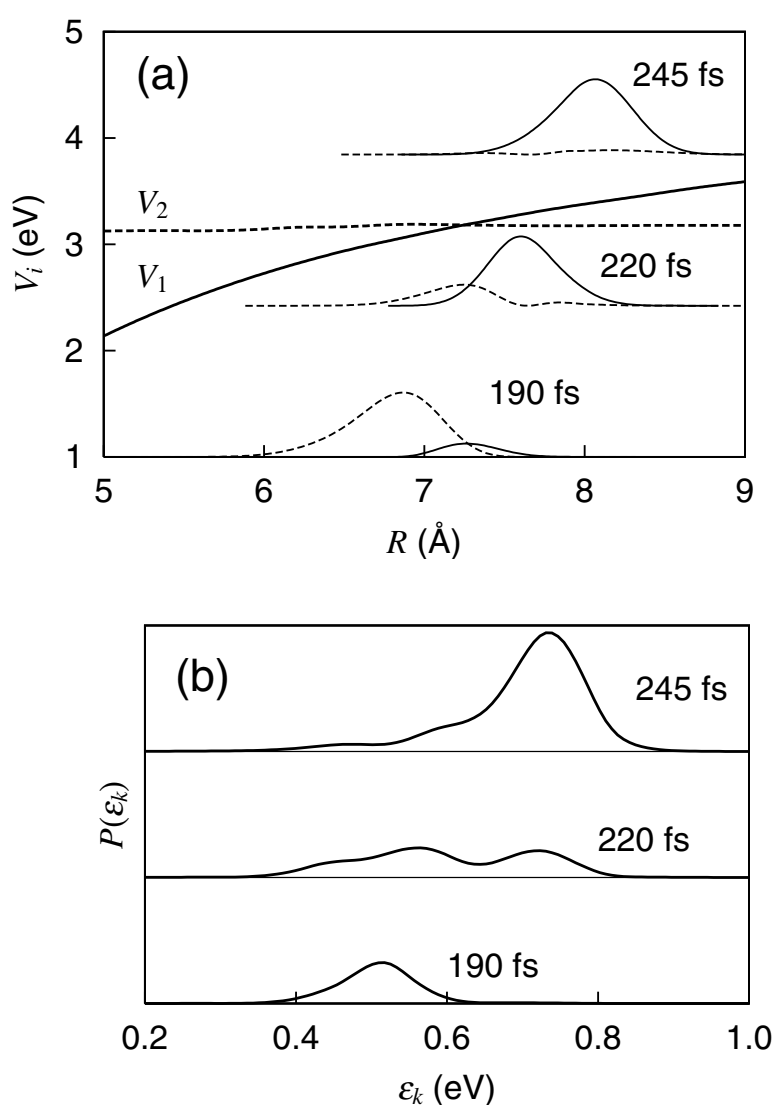

FIG. 3. Representative wave packet snapshots on the diabatic curves (a) and corresponding photoelectron energy distributions (b) as the wave packet passes through the nonadiabatic crossing. The vertical positioning of the wave packet snapshots bears no relationship to the energy axis. The vertical scales for the wave packets (a) and photoionization signals (b) are the same in Figs. 3-5, unless otherwise noted.

of $\sim 0.5 \mathrm{eV}$. As the wave packet is transferred to the $V_{1}$ curve (220 and $245 \mathrm{fs}$ ), it is forced to move uphill and slow, causing the photoelectron energies to increase to $\sim 0.7 \mathrm{eV}$. In the present case the difference in the nature of the potential curves (neutral vs Coulombic) enables a clear observation of the vibrational wave packet transferring from one diabatic electronic state to another.

Figure 4 depicts a case in which the nonadiabatic splitting of the wave packet produces a three-peaked signal. Figure 4(a) shows wave packet snapshots between 680 and 820 fs and 4(b) the corresponding photoelectron spectra. As in the previous example, ionization from the $V_{2}$ curve results in photoelectron kinetic energies of $\sim 0.5 \mathrm{eV}$ while ionization from the $V_{1}$ curve to the right of the crossing yields photoelectrons with $\sim 0.7 \mathrm{eV}$, directly manifesting the transfer and bifurcation of the wave packet between the diabatic states. A novel feature of the present case is a low-energy signal seen at $\sim 0.2 \mathrm{eV}$, which arises from the packet component that has evolved onto the $V_{1}$ state to the left of the crossing. Because of a large photoionization amplitude in the region near $6 \AA$, this small $V_{1}$ component caused by bifurcation of the wave packet at the crossing is observable in the spectrum.

Our final example is observation of wave packets with different histories after the second passage through the crossing point at about 700 fs. Figure 5 shows wave packet snapshots and photoelectron spectra in the time range 1090-1170 fs, corresponding to the third passage through the crossing point. The small packet on $V_{1}$, which by $1170 \mathrm{fs}$ is located near the right-hand turning point (9$10 \AA$ ), has been evolving on the $V_{1}$ curve, while the larger one is transferring from the $V_{2}$ curve, a process nearly over by 1170 fs. The photoelectron signals between 0.4 and $0.8 \mathrm{eV}$ again represent the larger wave packet bifurcated between the $V_{2}$ and $V_{1}$ curves, as in Fig. 3. Because of the large photoionization amplitude near the righthand turning point on $V_{1}$, ionization of the smaller packet in this region is also observable at $\sim 1.0 \mathrm{eV}$. Thus a threepeaked distribution becomes clearly visible at $1140 \mathrm{fs}$, but one that is completely different in nature from that observed at 735 fs in Fig. 4.

In this Letter we have shown that the passage and bifurcation of a wave packet through the avoided crossing can be observed as a splitting in the photoelectron energy signal. The key to making the effect more readily observable is that both the potential (and thus photoelectron) energy and the ionization amplitude should vary strongly
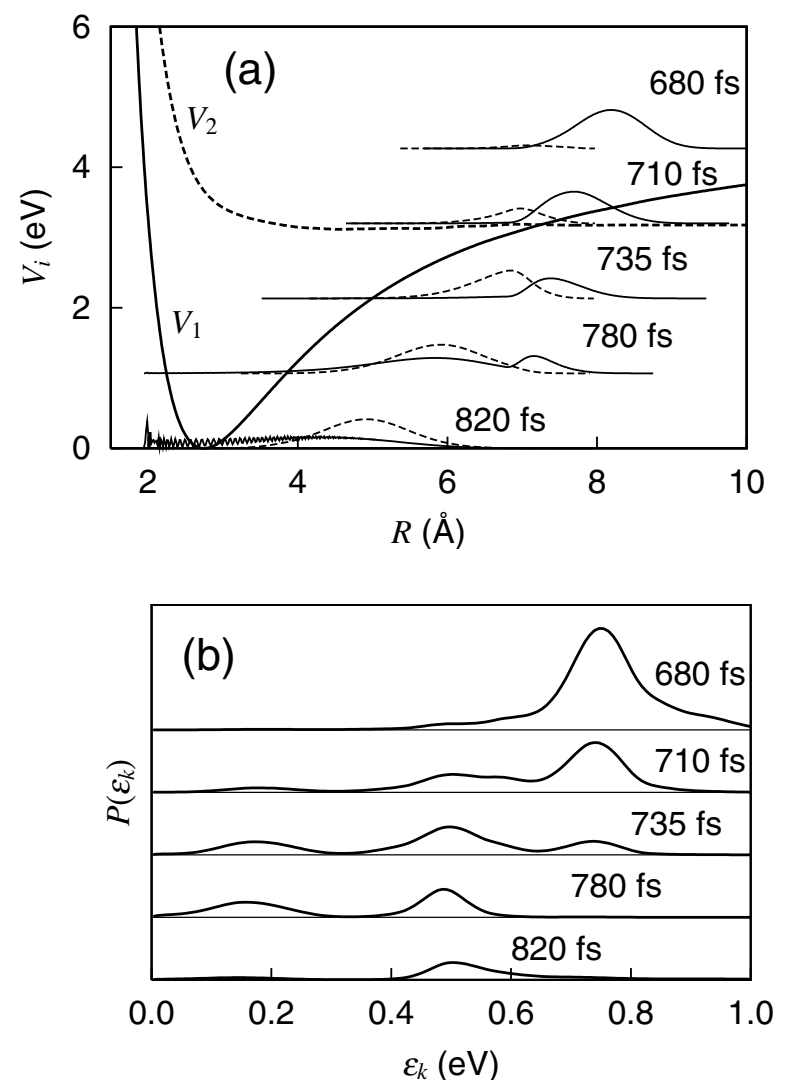

FIG. 4. Snapshots of the representative wave packets (a) and corresponding photoelectron signals (b) as in Fig. 3. Wave packets on $V_{1}$ at 780 and 820 fs magnified 10 times for clarity. 

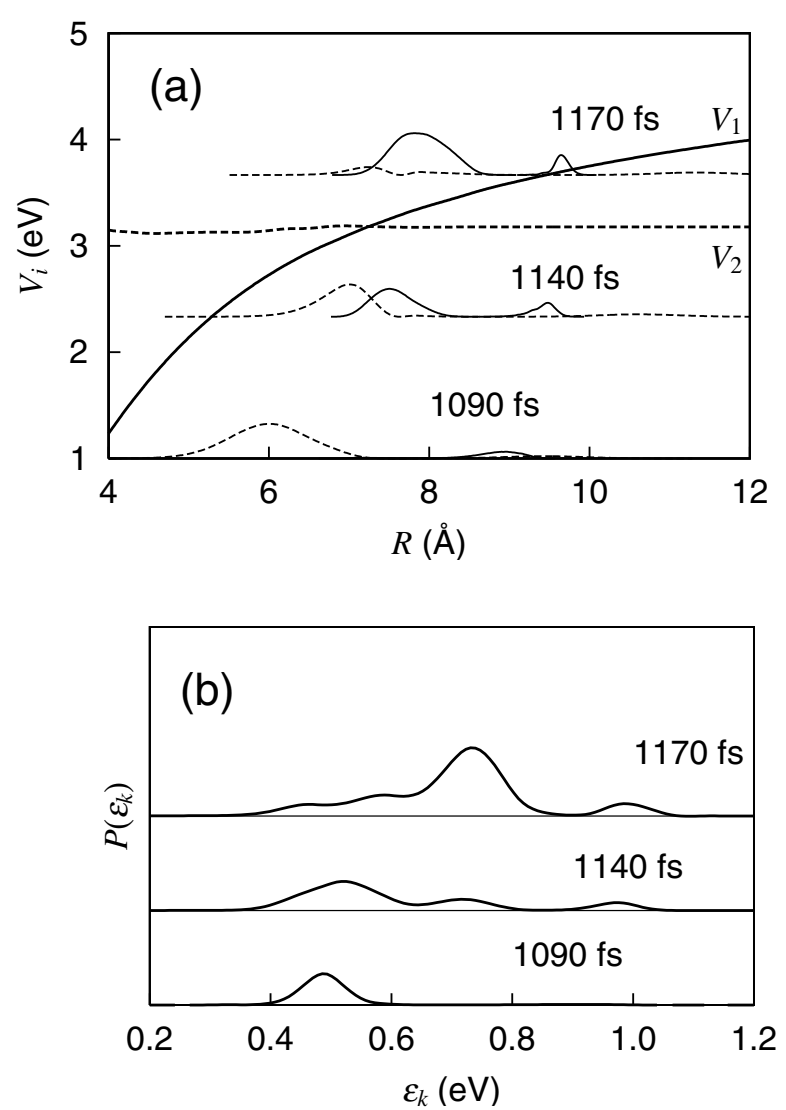

FIG. 5. Snapshots of the representative wave packets (a) and corresponding photoelectron signals (b) as in Fig. 3. The leading crest on $V_{1}$ is magnified 20 times for clarity.

with position on at least one of the potential curves involved. We note that the results presented are for a particular, favorable molecular orientation (molecular axis perpendicular to the probe pulse polarization); other orientations may be preferable in other systems. Although in practice the molecules will have a distribution of orientations, the pump pulse will produce a fairly aligned distribution; moreover, molecular rotation can be neglected during the short time between pump and probe. Finally, conical intersections are ubiquitous in polyatomics [25] and result in much more complex bifurcation dynamics than in the simpler case of an avoided crossing in a diatomic system studied here. The formulation and computational implementation employed here are being extended to higher dimensions and will be used in studies of pump-probe photoelectron spectra at conical intersections in a few simple systems.

This work has been supported in part by a Grant-inAid for the 21st Century COE Program for Frontiers in Fundamental Chemistry from the Ministry of Education, Culture, Sports, Science and Technology (Japan), by the Mitsubishi Foundation, and by the National Science Foundation (U.S.A.).
*Electronic address: kaztak@mns2.c.u-tokyo.ac.jp

[1] See H. Nakamura, Nonadiabatic Transition (World Scientific, Singapore, 2002) for the latest review.

[2] M.S. Child, Semiclassical Mechanics with Molecular Applications (Oxford University, New York, 1991).

[3] T. S. Rose, M. J. Rosker, and A. H. Zewail, J. Chem. Phys. 88, 6672 (1988); 91, 7415 (1989).

[4] P. Cong, G. Roberts, J. L. Herek, A. Mohktari, and A. H. Zewail, J. Phys. Chem. 100, 7832 (1996).

[5] A. Tanaka, J. Phys. A 29, 5475 (1996); Phys. Rev. Lett. 80, 1414 (1998).

[6] H. Köppel, W. Domcke, and L. S. Cederbaum, Adv. Chem. Phys. 57, 59 (1984); D. M. Leitner, H. Köppel, and L. S. Cederbaum, J. Chem. Phys. 104, 434 (1996).

[7] H. Fujisaki and K. Takatsuka, Phys. Rev. E 63, 066221 (2001); J. Chem. Phys. 114, 3497 (2001).

[8] H. Higuchi and K. Takatsuka, Phys. Rev. E 66, 035203(R) (2002).

[9] A. Assion, M. Geisler, J. Helbing, V. Seyfried, and T. Baumert, Phys. Rev. A 54, R4605 (1996).

[10] V. Blanchet, M. Z. Zgierski, T. Seideman, and A. Stolow, Nature (London) 401, 52 (1999).

[11] J. A. Davies, J. E. LeClaire, R. E. Continetti, and C.C. Hayden, J. Chem. Phys. 111, 1 (1999).

[12] T. Suzuki, L. Wang, and K. Kohguchi, J. Chem. Phys. 111, 4859 (1999).

[13] Y. Arasaki, K. Takatsuka, K. Wang, and V. McKoy, J. Chem. Phys. 112, 8871 (2000).

[14] S. Lochbrunner, T. Schultz, M. Schmitt, J. P. Shaffer, M. Z. Zgierski, and A. Stolow, J. Chem. Phys. 114, 2519 (2001).

[15] C. Jouvet, S. Martrenchard, D. Solgadi, C. DedonderLardeux, M. Mons, G. Grégoire, I. Dimicoli, F. Piuzzi, J. P. Visticot, J. M. Mestdagh, P. D’Oliveira, P. Meynadier, and M. Perdrix, J. Phys. Chem. A 101, 2555 (1997).

[16] G. Grégoire, M. Mons, I. Dimicoli, F. Piuzzi, E. Charron, C. Dedonder-Lardeux, C. Jouvet, S. Martrenchard, D. Solgadi, and A. Suzor-Weiner, Eur. Phys. J. D 1, 187 (1998).

[17] V. Engel and H. Metiu, J. Chem. Phys. 90, 6116 (1989).

[18] H. Kono and Y. Fujimura, Chem. Phys. Lett. 184, 497 (1991).

[19] M. Braun, C. Meier, and V. Engel, J. Chem. Phys. 105, 530 (1996).

[20] E. Charron and A. Suzor-Weiner, J. Chem. Phys. 108, 3922 (1998).

[21] K. Takatsuka, Y. Arasaki, K. Wang, and V. McKoy, Faraday Discuss. 115, 1 (2000).

[22] A. B. Alekseyev, H.-P. Liebermann, R. J. Buenker, N. Balakrishnan, H. R. Sadeghpour, S.T. Cornett, and M. J. Cavagnero, J. Chem. Phys. 113, 1514 (2000).

[23] W. Kim, W. Qin, D. G. McCoy, and L.W. Torop, Chem. Phys. 264, 401 (2001).

[24] Y. Arasaki, K. Takatsuka, K. Wang, and V. McKoy (to be published).

[25] See, for example, Chr. Cattarius, G. A. Worth, H.-D. Meyer, and L. S. Cederbaum, J. Chem. Phys. 115, 2088 (2001). 